Methods We assayed specific IgE sensitisation in 300 bakers employed by one of two large UK supermarkets who, at routine health surveillance, had declared work related upper or lower respiratory symptoms. Sensitisation was determined using radioallergosorbent assay to enzymes contained within the specific 'improver' mix used by the employing supermarket; each mix contained eight individual enzymes which were not necessarily common to both supermarkets.

Results Bakers were sensitised to each of the individual 'improver' enzymes with a prevalence ranging from $1.8 \%$ to $23.9 \%$; the frequency did not appear to be associated with the quantity of enzyme incorporated in the mix. Sensitisation was far more likely if a baker was sensitised also to either flour or fungal alpha amylase; but a small proportion (5\%) of bakers who were sensitised to neither flour nor fungal alpha amylase had specific IgE to one or more of the 'improver mix' enzymes. Conclusions Bakers working in UK supermarket bakeries can become sensitised to improver enzymes other than fungal alpha amylase. The clinical significance of this remains unclear but the message is important both in the diagnosis of bakers with workrelated respiratory symptoms and in any programme of immunological surveillance.

\section{S6 1 THE ROLE OF INDIVIDUALLY VENTILATED CAGES IN PREVENTION OF LABORATORY ANIMAL ALLERGY: A PROOF OF CONCEPT STUDY}

${ }^{1} \mathrm{JR}$ Feary, ${ }^{1} \mathrm{Z}$ Lightfoot, ${ }^{1} \mathrm{~B}$ Fltzgerald, ${ }^{2} \mathrm{~S}$ Schofield, ${ }^{2} \mathrm{M}$ Jones, ${ }^{2} \mathrm{P}$ Cullinan. ${ }^{1}$ Royal Brompton and Harefield NHS Foundation Trust, London, UK; ${ }^{2}$ Imperial College, London, UK

\subsection{6/thoraxjnl-2014-206260.12}

Introduction and objectives At least 12000 people work with laboratory animals in the UK. Approximately $15 \%$ of exposed employees develop specific IgE sensitisation and 10\% clinical symptoms of laboratory animal allergy (LAA), a form of occupational asthma. Individually ventilated cages (IVCs) are increasingly replacing conventional open cages (primarily to protect mice from external infection) and whilst this can be associated with lower levels of ambient aeroallergen levels no corresponding reduction in the incidence of LAA is apparent. The SPIRAL

\section{Abstract S6 Table 1 Baseline characteristics SPIRAL study} participants to date and preliminary results

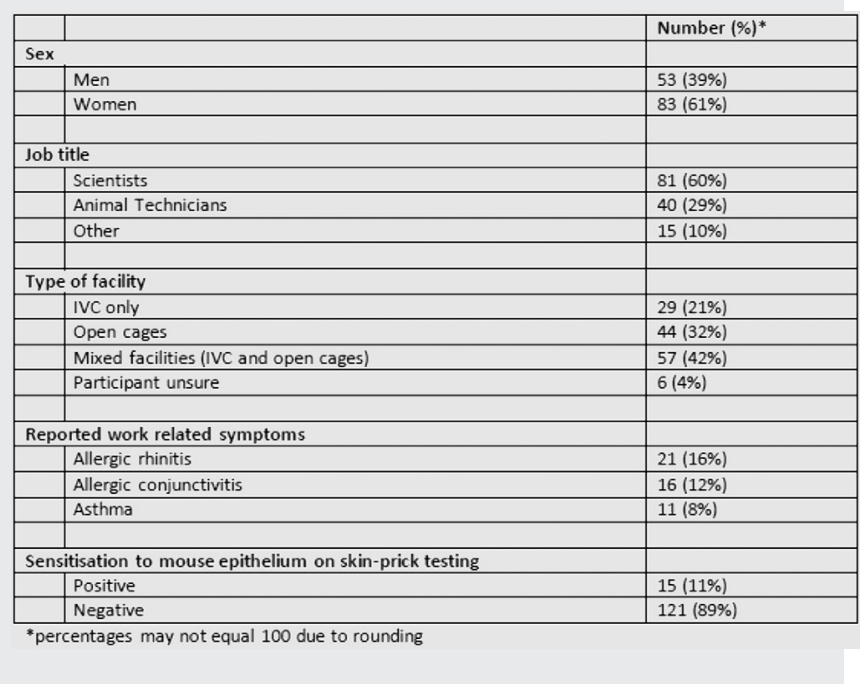

(Safe Practice In Reducing Allergy in Laboratories) study is a large multi-centred study designed to increase understanding of the complex association between workplace exposure to mouse allergens and development of sensitisation, and to evaluate the risk of working with mice today.

Methods A cross-sectional study of animal workers at seven UK medical research institutions is in progress. We aim to recruit 250 people working in IVC-only facilities and 160 people working in mixed facilities; our primary outcome is a comparison of prevalence of sensitisation to Mus $\mathrm{m} 1$ (mouse urinary antigen) between these two groups. Participants are invited to complete a detailed online questionnaire about work tasks and practices. Skin-prick tests to common aeroallergens and various animal proteins are performed and blood samples analysed for serum specific IgE to Mus $\mathrm{m}$ 1. Aeroallergen sampling for particular matter and Mus $\mathrm{m} 1$ is undertaken concurrently to provide additional information about potential exposures. Recently employed individuals are invited to participate in a cohort study to determine incidence rates of laboratory animal allergy.

Results 136 individuals have been recruited to date; Table 1 shows their demographics and preliminary immunology results. Prevalence of allergic symptoms and sensitisation is similar to that anticipated.

Conclusion The SPIRAL study is the largest, most detailed study of LAA to be carried out. It aims to increase understanding of the relationship between allergen exposure and risk of sensitisation with the goal of significantly reducing the incidence of LAA. The results will be used to develop a "Code of best working practices" for facilities using IVC systems, nationally and further afield.

\section{New approaches to the management of ILD}

\section{S7 NITROFURANTOIN LUNG TOXICITY - ARE STEROIDS USEFUL?}

${ }^{1} \mathrm{ADL}$ Marshall, ${ }^{2} \mathrm{HK}$ Bayes, ${ }^{3} \mathrm{OJ}$ Dempsey. ${ }^{1}$ Department of Respiratory Medicine, Glasgow Royal Infirmary, Glasgow, UK; ${ }^{2}$ Institute of Infection, Immunity and Inflammation, University of Glasgow, Glasgow, UK; ${ }^{3}$ Chest Clinic C, Aberdeen Royal Infirmary, Aberdeen, UK

\subsection{6/thoraxjnl-2014-206260.13}

Background Recurrent urinary tract infections are common and current UK guidelines advocate prophylaxis with nitrofurantoin in selected patients ${ }^{1}$. Nitrofurantoin-induced pulmonary toxicity ("Nitrofurantoin lung" or NL) is uncommon but can result in progressive respiratory failure. Locally we have observed a rise in NL. Pulmonary toxicity necessitates cessation of nitrofurantoin, however the utility of additional corticosteroid therapy remains controversial ${ }^{2}$. We examined a detailed case series to ascertain the effect of drug cessation alone and addition of oral corticosteroids in NL.

Methods Using a local Interstitial Lung Disease database, we retrospectively identified patients who had presented with NL between 2009-2013. Patient demographics, imaging, pulmonary function and prescribing data were accessed. Local and national nitrofurantoin prescribing rates were also reviewed.

Results Scottish prescribing data demonstrated increased community nitrofurantoin prescriptions from 3.4 to 11 items/1000 patients from 2008-2012. Our database identified 13 NL cases (93\% female, mean age 74 years, range 63-83). All had a history of chronic cystitis and presented with chronic NL. Cumulative 